
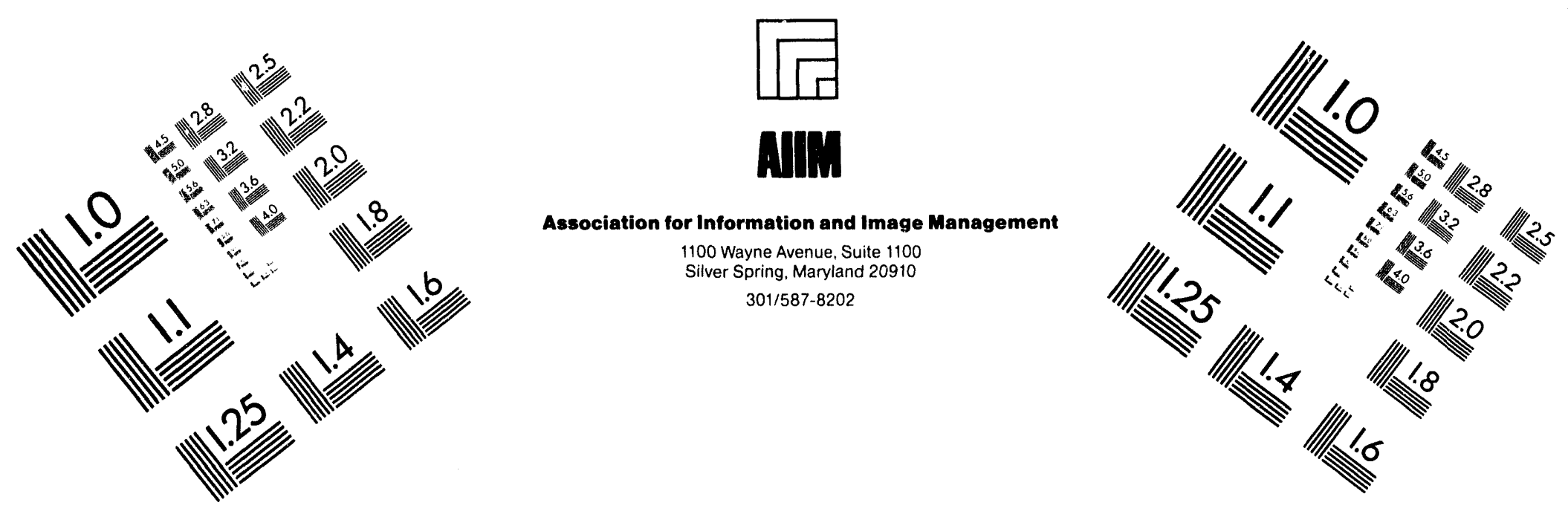

Centimeter

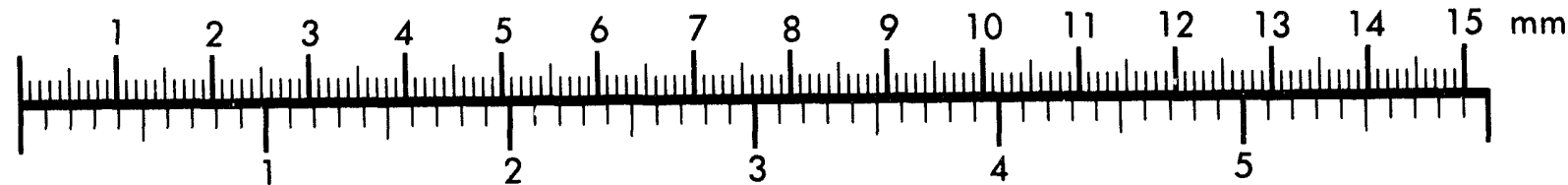
Inches
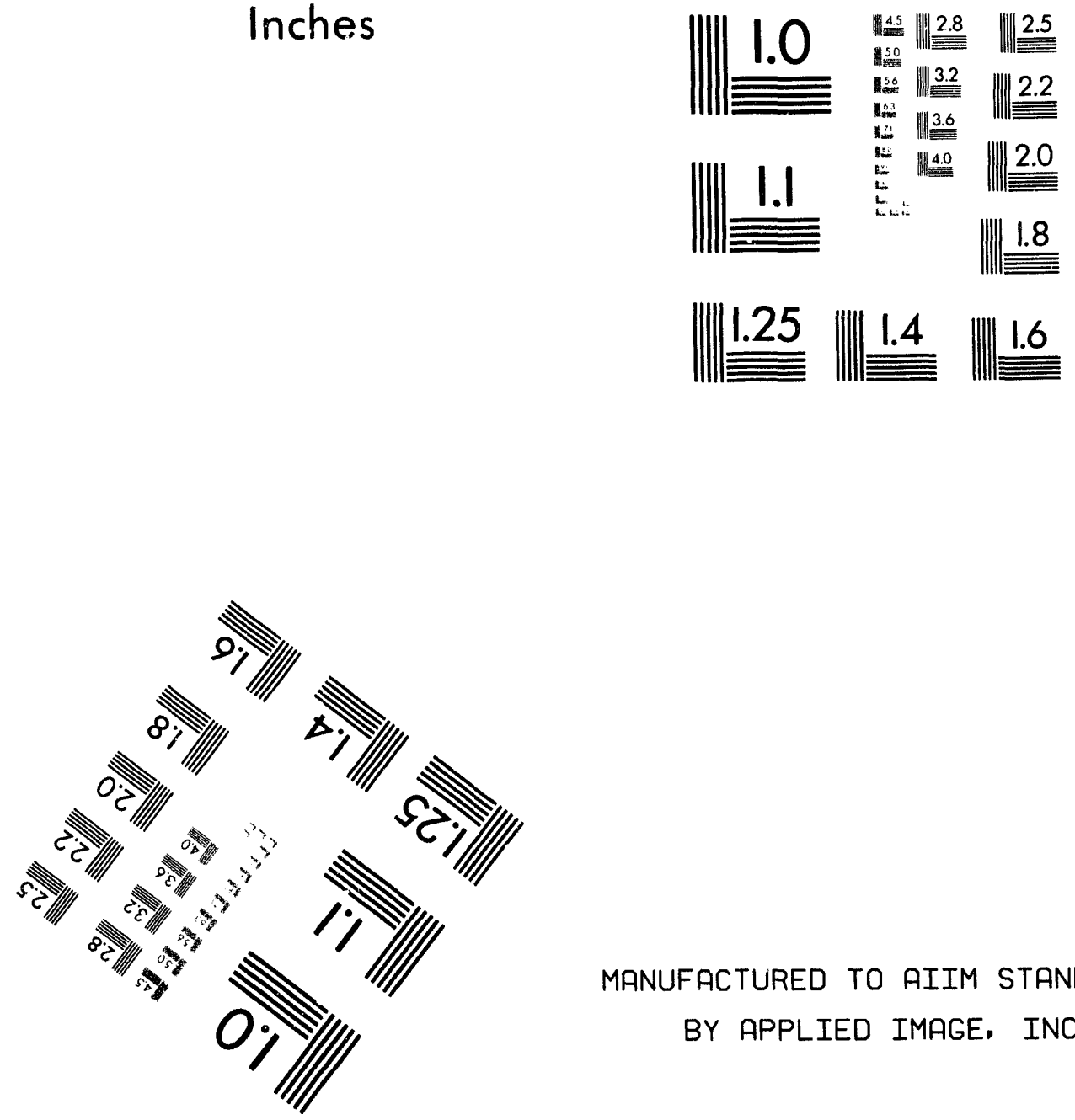

MANUFACTURED TO AIIM STANDARDS

BY APPLIED IMAGE, INC.

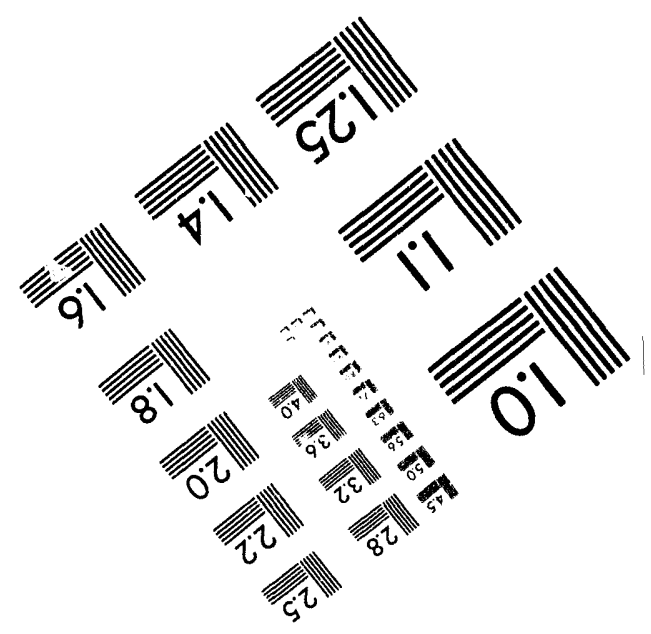



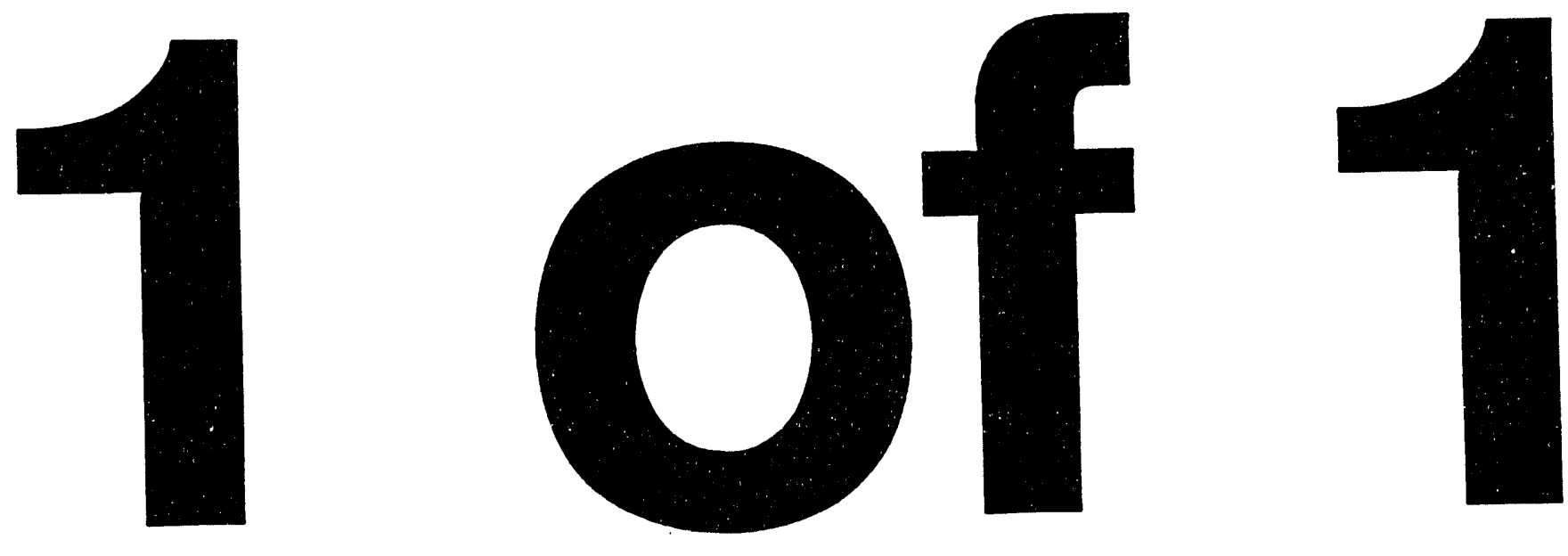


$$
\begin{aligned}
& \text { DOEIPC |90303-IT' } \\
& \text { Qu. Ter C Teeh Repont } \\
& 12131192 \\
& 45192
\end{aligned}
$$

DE. FG.22.90PC90303

$\mathrm{Ju}, 1$

\title{
Effect of the Bioemulsifier Emulsan on Naphthalene Mineralization from Coal Tar in Aqueous Systems
}

\author{
KAREN L. SKUBAL AND RICHARD G. LUTHY* \\ Department of Civil Engineering \\ Carnegie Mellon University \\ Pittsturgh, Pennsylvania 15213
}

\section{DISCLAIMER}

This report was prepared as an account of work sponsored by an agency of the United States Government. Neither the United States Government nor any agency thereof, nor any of their employees, makes any warranty, express or implied, or assumes any legal liability or responsibility for the accuracy, completeness, or usefulness of any information, apparatus, product, or process disclosed, or represents that its use would not infringe privately owned rights. Reference herein to any specific commercial product, process, or service by trade name, trademark, manufacturer, or otherwise does not necessarily constitute or imply its endorsement, recommendation, or favoring by the United States Government or any agency thereof. The views and opinions of authors expressed herein do not necessarily state or reflect those of the United States Government or any agency thereof.

* corresponding author (phone 412/268-2940, fax 412/268-7813)

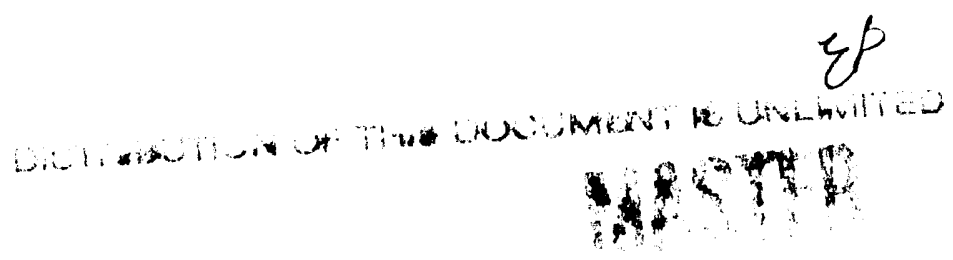




\begin{abstract}
Coal tar in aerobic aqueous systems was treated with purified emulsan, the anionic heteropolysaccharide bioemulsifier produced by Acinetobacter calcoaceticus RA G-1; with inocula of various concentrations of stationary phase RAG-1 cells; or with cell-free broth from stationary phase RAG-1 cultures. Naphthalene mineralization by a mixed PAH-degrading population was measured by recovering ${ }^{14} \mathrm{CO}_{2}$ evolved during biotransformation of the $\left[{ }^{14} \mathrm{C}\right]$ naphthalene-labeled coal tar. There was no evidence of naphthalene mineralization by RAG-1 cells alone. The addition of emulsan, RAG-1 inocula, or cell-free broth to systems containing the PAH-degrading population did not significantly affect naphthalene mineralization in any of the systems tested. Coal tar in these experiments was present either as a free dense nonaqueous phase liquid (DNAPL), or as DNAPL imbibed into microporous silica particles. Emulsification of the tar was not observed in either case. The presence or absence of microporous silica did not affect the extent or rate of naphthalene mineralization, nor did the concentration of RAG-1 inocula or the amount of broth added. The addition of cell-free broth, emulsan, or RAG-1 cells late in the experiments did not yield significantly different results compared to initial addition of these substances. Thus, emulsan and related fractions from RAG-1 cultures were ineffective in altering naphthalene mineralization in this study.
\end{abstract}

KEYWORDS - Emulsan

Mineralization
Coal tar

Naphthalene
Bioemulsifier 


\section{INTRODUCTION}

Coal tar is a dense nonaqueous phase liquid (DNAPL) by-product of coal carbonization. During carbonization, coal is exposed to high temperatures and may be reacted variously to produce a combustible gas, hydrocarbon liquids, coke, or tar [1]. Coal tar is a complex mixture, with over $\mathbf{4 0 0}$ compounds having been identified in various tars [2]. However, most coal tars consist primarily of highly hydrophobic aromatic compounds ranging in size from single-ring benzene structures to complex, multi-ring polycyclic aromatic (PAH) compounds [3].

The accidental release of coal tar into the environment and the subsequent penetration of tar into the subsurface have resulted in soil and groundwater contamination by PAH compounds and other coal tar constituents at certain former manufactured gas plant sites. PAH compounds have low aqueous solubilities, which may affect the rate of their biodegradation in soil/water systems [4], and they exhibit the ability to persist and bioaccumulate in nature [5]. Aerobic biodegradation of 2-and 3-ring PAH compounds has been widely observed, al though the transformation rate for 2-ring substrates, such as naphthalene, is typically greater than that for 3-ring compounds, i.e., anthracene or phenanthrene [6]. The PAH-degrading organisms used in this study have tentatively been identified as a consortium of Pseudomonas species. Pseudomonads are widespread in soil and water and possess enzyme systems capable of transforming a broad range of natural organic compounds and xenobiotics, even those with very different structures [7].

One of the most important factors affecting biodegradation is the availability of substrate to microorganisms. Three modes of hydrocarbon transport to bacterial cells have been postulated [8]. The first is the interaction of bacteria with hydrocarbon dissolved in the aqueous phase. The second is the interaction of cells with solubilized or pseudosolubilized hydrocarbon droplets much smaller than the cells, in which case hydrocarbon microdroplets attach to cells rather than vice versa. The third is direct contact between cells and large hydrocarbon droplets, with uptake thought to occur through diffusion or active transport by the cells. Substrate availability is especially significant for bioremediation of highly hydrophobic mixtures such as coal tar, which may preferentially partition into soil or other hydrophobic phases present, or remain underground 
as a dense nonaqueous phase liquid (DNAPL) [9]. Substrate availability from coal tar is affected by the release of solute from the coal tar phase, and by solute partitioning into the soil phase, which attenuates the transport of hydrophobic organic compounds through the subsurface. These processes may limit the fraction of substrate present in the bulk aqueous phase, where microbial uptake is thought to predominate $[10,11]$. This in tum may decrease the biodegradation rate compared to tar-free or soil-free systems with the same quantity of contaminant.

\section{Biosurfactants and bioemulsifiers}

Biosurfactants and bioemulsifiers are two classes of compounds that could prove useful in counteracting substrate insolubility or sorption, and enhancing hydrocarbon biodegradation in the aqueous phase. The addition of surfactants to soil-water systems has been proposed as a means of desorbing hydrophobic contaminants from soil and solubilizing them through incorporation into surfactant micelles [12]. One disadvantage of adding synthetic surfactants in situ may be possible toxicity or inhibition of microorganisms responsible for biodegradation, or use of the surfactant as substrate in preference to the target hydrocarbon. However, the production of biosurfactants and bicemulsifiers by hydrocarbon-utilizing bacteria is well documented [8], and toxicity or inhibition by these substances may be less of a concern [13]. The increase in surface area of the hydrocarbon-water interface resulting from hydrocarbon emulsification by these bioproducts could enhance the availability of hydrophobic contaminants to bacteria and promote biodegradation in the aqueous medium.

Biosurfactants are classed as glycolipids, phospholipids, neutral lipids, fatty acids, polysaccharides and lipopeptides [14]. They function by lowering the interfacial tension between water and hydrocarbon compounds, thus permitting emulsification of the hydrocarbons into the water phase [13]. Bioemulsifiers, on the other hand, are usually large biopolymers such as polysaccharides that do not reduce interfacial tension appreciably $[13,14]$ but instead bind tightly to

the water-oil interface and prevent oil droplet coalescence [13]. Emulsan, a nontoxic bioemulsifier 
produced by the gram-negative bacteria Acinetobacter calcoaceticus RAG-1 (ATCC 31012), was used in this study.

Emulsan is the extracellular form of a polyanionic, cell-associated heteropolysaccharide (molecular weight $\sim 10^{6}$ ) produced by the RAG-1 organism [15,16]. Although emulsan exhibits specificity regarding the hydrocarbons it emulsifies, it is excreted into the growth medium even when RAG-1 is grown on water-soluble substrates such as ethanol or acetate [15]. The bioemulsifier's amphipathic nature is due to its hydrophilic polysaccharide backbone with covalently-linked fatty acid ester side chains, which comprise approximately $15 \%$ of the polymer by weight [15]. The polysaccharide portion of the molecule contains the principal sites responsible for its emulsifying activity and is composed of two major sugars, N-acetyl-D-galactosamine and an $\mathrm{N}$-acetyl hexosamine uronic acid [16]. D-glucose is a minor sugar component, comprising $5.2 \%$ of the molecule by weight [17]. Emulsan also contains 15 to $20 \%$ ( $w t w t)$ noncovalently bound protein [16], which is not necessary for emulsification but does enhance the biopolymer's activity. An approximate structure has been shown for emulsan [13].

The production of emulsan by RAG-1 and the bioemulsifier's role in RAG-1 growth and adherence are both growth-associated [18]. Emulsan initially accumulates on the RAG-1 cell surface as a cell-associated minicapsule and is released as an active emulsifier during conditions of unbalanced growth or as the cells approach stationary phase $[16,19]$. Cell-associaied emulsan capsules create a hydrophilic environment at the RAG-1 cell surface and preciude adherence to hydrocarbons, while cell-free emulsan orients itself at the hydrocarbon-water interface to form a hydrophilic film around oil droplets, effecting emulsification [20]. The stability and physical characteristics (i.e. droplet size and emulsan film thickness) of emulsions stabilized by emulsan depend upon the relative, not absolute, concentrations of emulsan and hydrocarbon [21], indicating that the bioemulsifier interacts directly with hydrocarbon rather than simply al tering surface tension [18].

Emulsan is optimally active when a mixture of aromatic and aliphatic hydrocarbons is present [22]. For example, hexadecane stimulates emulsification of many PAH compounds that by 
themselves are not emulsified by emulsan, while aromatic compounds including 1methylnaphthalene, 2-methylnaphthalene, toluene, and p-xylene induce emulsification of aliphatic compounds. Furthermore, 1-methylnaphthalene was found to enhance slightly the emulsification of naphthalene, phenanthrene, and biphenyl by emulsan, despite the fact that none of these compounds alone is emulsified [22]. Because 1-methylnaphthalene was a major identifiable component of the coal tar used in this research (Table 1), the potential existed for it to affect naphthalene emulsification and mineralization in the systems being studied.

Emulsan's role in hydrocarbon biodegradation by species other than RAG-1 is unclear but is probably substrate- and species-specific. Several studies have examined the bioemulsifier's effect on hydrocarbon biodegradation by other mictoorganisms. One study [23] found that marine microcosms containing fuel oil and $0.015 \%$ emulsan showed increased microbial respiration and increased numbers of hydrocarbon-degrading bacteria compared to systems without emulsan, although increased respiration may have resulted in part from emulsan degradation. Another study [20] used $0.005 \%$ emulsan with both pure and mixed bacterial cultures. Emulsan was found to inhibit alkane mineralization (n-hexadecane and pristane) by six pure cultures. Aromatic mineralization (phenanthrene and carbazole) by two pure cultures of PAH-degrading organisms was either slightly stimulated or unaffected by emulsan, while aromatic mineralization in crude oil by a mixed population was inhibited. Clearly, emulsan's effect on hydrocarbon biodegradation under laboratory conditions depends upon the microorganisms and the hydrocarbon substrate being considered.

Practical use of emulsan in science and industry is apparently still limited. However, it has been suggested [20] that emulsan's ability to stabilize emulsions of hydrocarbons in water could prove useful in the petroleum industry, where it could be used to form heavy oil-water emulsions for viscosity reduction during pipeline transport. Emulsan may also find a place in microbiological studies as an antiadherence agent for desorbing bacteria that adhere vigorously to hydrophobic surfaces [24]. 
This study assesses whether the bioemulsifier emulsan or related bioproducts affect naphthalene mineralization from coal tar in aerobic aqueous systems. Batch systems containing coal tar either as a free-phase DNAPL, or as DNAPL imbibed in microporous silica were studied. Purified emulsan, RAG-1 organisms, or cell-free RAG-1 culture medium was added to these systems. The results of this work intend to show whether emulsan or related substances are able to significantly enhance naphthalene mineralization by PAH-degrading soil bacteria under laboratory conditions.

\section{MATERIALS AND METHODS}

\section{Organisms and culture conditions}

The naphthalene-degrading culture used in this study consisted of a mixed population of PAH-degrading organisms that had been isolated from contaminated soils and wastes [9]. The bioemulsifier-producing strain used was Acinetobacter calcoaceticus RAG-1, which produces emulsan, an extracellular amphipathic heteropolysaccharide bioemulsifier [16].

The PAH-degrading organisms were grown aerobically in autoclaved BOD dilution water [25] with crystalline naphthalene (purity $>98 \%$, Fisher Scientific, Fair Lawn, NJ) as the sole carbon source. The BOD dilution water was amended with fifty times the suggested $\mathrm{NH}_{4} \mathrm{Cl}$ concentration to prevent $\mathrm{NH}_{3}-\mathrm{N}$-limited growth conditions. Cultures were kept at room temperature on an orbital shaker (Lab Line Orbital Shaker, Model 3590, VWR Scientific Co.) and shaken continuously at $50 \mathrm{rpm}$.

PAH-degrading orgarisms were enumerated by spreading $0.2 \mathrm{~mL}$ aliquots of $10^{-4}$ to $10^{-7}$ culture dilutions on duplicate agar plates. The plates were prepared using high-nitrogen BOD dilution water with $1.5 \%$ (w/w) agar (Difco Bacto-Agar, Difco Laboratories, Detroit, MI). After the agar was spread with culture, the petri dishes were inverted and crystalline naphthalene was placed on the inside of the lid. Vapors from the solid naphthalene entered the agar, providing a carbon source for the organisms. After four days or longer, colonies were identifiable on the agar surface. The PAH-degrading bacteria typically grew to $\sim 10^{7} \mathrm{CFU} / \mathrm{mL}$. 
The bicemulsifier-producing RAG-1 organisms were grown on an ethanol-minimal salts medium containing (per liter of water): $\mathrm{K}_{2} \mathrm{HPO}_{4} \cdot 3 \mathrm{H}_{2} \mathrm{O}, 22.2 \mathrm{~g} ; \mathrm{KH}_{2} \mathrm{PO}_{4}, 7.26 \mathrm{~g} ; \mathrm{MgSO}_{4}$. $\mathrm{TH}_{2} \mathrm{O}, 0.2 \mathrm{~g}$; $\left(\mathrm{NH}_{4}\right)_{2} \mathrm{SO}_{4}, 4 \mathrm{~g}$; ethanol, $20 \mathrm{~g}$ [15]. The cultures were maintained in flasks at room temperature and were mixed on electromagnetic stir plates (15-point stirrer, Cole Palmer Instruments, Chicago, IL) set to stir for sixty seconds every five minutes. These organisms were enumerated by spreading $0.2 \mathrm{~mL}$ aliquots of $10^{-5}$ to $10^{-9}$ culture dilutions on nutrient agar (BBL Nutrient Agar, Becton Dickinson Microbiology Systems, Cockeysville, MD). Cream-colored colonies appeared within $3-4$ days, and the RAG-1 bacteria typically grew to $\sim 10^{9} \mathrm{CFU} / \mathrm{mL}$ in liquid medium. The medium changed color from clear to opaque yellow during the two weeks following inoculation, and vigorous shaking of the culture resulted in the formation of froth.

\section{Mineralization lests}

Aerobic biodegradation of naphthalene was studied in closed systems using radiolabeling techniques. The systems were contained in $250-\mathrm{mL}$ biometer flasks (Bellco Glass, Inc., Vineland, NJ). The flask side of the apparatus held bacteria in a liquid medium, while the glass side tube contained $5 \mathrm{~mL}$ of $2 \mathrm{M} \mathrm{NaOH}$ solution, which was used to capture $\mathrm{CO}_{2}$ evolved by the PAHdegrading organisms. Both the flask and side tube openings were sealed with Neoprene stoppers, which were removed only for sampling and weekly aeration with oxygen.

Mineralization tests were performed using coal tar as the sole carbon source. The coal tar used was obtained from the subsurface at the site of a former manufactured gas plant site in Stroudsburg, PA [26]. A partial composition of Stroudsburg coal tar is shown in Table 1. Prior to use, the coal tar was radiolabeled by adding a measured volume of naphthalene-1-14C (Sigma Chemical Co., St. Louis, MO) stock solution. Approximately $0.9-2 \mu \mathrm{Ci}$ of $\left.{ }^{14} \mathrm{C}\right]$ naphthalene, corresponding to $\sim 2 \times 10^{6}-4 \times 10^{6} \mathrm{dpm}$, was added per milliliter of coal tar.

Radiolabeled coal tar was added to biometer flasks either as a separate liquid phase or imbibed in microporous silicon dioxide, which was used to simulate microporous soil particle aggregates. The silica had a median particle diameter of $236 \mu \mathrm{m}$, a pore diameter of $\sim 200 \AA$ and a porosity of 0.78 (IMPAQ RG 20150Si granular chromatographic silica, PQ Chromatography 
Products, Valley Forge, PA). The silica was pretreated by heating in a furnace at $800^{\circ} \mathrm{F}$ for two hours to cross-link surface hydroxide groups and slightly increase the material's hydrophobicity, thus enhancing its ability to imbibe and hold the coal tar. After cooling, five-gram portions of silica were weighed and placed in $50 \mathrm{~mL}$ Pyrex centrifuge tubes. One milliliter of radiolabeled coal tar was added to each tube, and the tubes were capped and mixed until the coal tar was evenly distributed among the silica granules. The tubes were placed on a rotator for 24 hours to ensure thorough mixing, then put aside for at least a week to allow the coal tar to be imbibed by the silica.

After a week or more, 5 gram samples of tarred silica were placed in biometer flasks with $50 \mathrm{~mL}$ of sterile nitrogen-enriched BOD dilution water. The $\mathrm{pH}$ was adjusted to 5.5-6.0 and readjusted the following day if necessary. Systems containing coal tar without silica were treated similarly and contained $1 \mathrm{~mL}$ of labeled tar in $50 \mathrm{~mL}$ of BOD dilution water. All biometer tests were performed at least in duplicate.

At the beginning of each experiment, biometer flasks were inoculated with PAH-degrading organisms, bioemulsifier-producing RAG-1 bacteria, or a combination of both. The inocula consisted of $2 \mathrm{~mL}$ aliquots of the respective cultures. PAH-degrading inocula contained $\sim 10^{7}$ CFU/mL, while RAG-1 inocula were used at approximately $10^{7}, 10^{8}, 10^{9}$, and $10^{10} \mathrm{CFU} / \mathrm{mL}$. Variations in the RAG-1 inoculum concentration were achieved either by diluting the original culture, or by centrifuging the culture and resuspending pelleted cells in a smaller volume of sterile BOD dilution water. Control flasks were not inoculated and were used to verify that napinthalene mineralization or volatilization did not occur in the absence of added microorganisms.

Some biometer flasks also initially received purified bioemulsifier (emulsan) or cell-free supernatant from bioemulsifier-producing cultures to assess whether substances produced by RAG-1 bacteria, but no longer associated with the cells, could affect mineralization by PAHdegrading organisms. Emulsan was supplied in powder form by Dr. Carol Litchfield (Keystone Environmental, Monroeville, PA) and used as received [23]. It was added to biometer flasks to give a final concentration of $0.015 \%$ by weight water [23]. Cell-free supernatant was obtained by centrifuging RAG-1 cultures for 20 minutes at $6500 \times g$ to pellet the cells. The supernatant was 
separated from the pellet, expressed through a $0.45 \mu \mathrm{m}$ Millipore filter, and added to biometer flasks in $10 \mathrm{~mL}$ aliquots.

The behavior of $\left[{ }^{14} \mathrm{C}\right]$ naphthalene was assumed to represent that of the unlabeled compound in biometer flasks. Naphthalene mineralization was tracked by measuring ${ }^{14} \mathrm{CO}_{2}$ that was released by PAH-degrading organisms and captured in the $\mathrm{NaOH}$ solution of the biometer flasks. During sampling, $0.5 \mathrm{~mL}$ of $\mathrm{NaOH}$ was removed in triplicate from each biometer and added to $10 \mathrm{~mL}$ of scintillation cocktail (Optifluor, Packard Instrument Co., Downers Grove, IL) in scintillation vials. After dark overnight storage to minimize chemiluminescence, the samples were counted for ${ }^{14} \mathrm{C}$ in a Beckman LS 5000 TD liquid scintillation counter with the H\# quench monitoring technique and automatic quench compensation [4]. Sodium hydroxide was replaced in the side arm of each biometer after every sampling interval.

\section{RESULTS}

\section{Substrate uptake}

Figure 1 [27] illustrates some of the processes envisioned to lead to microbial uptake and biodegradation of a PAH compound released from the coal tar DNAPL in the porous silica medium. Visual observations and scanning electron microscopy suggest that most of the DNAPL is present as a liquid within microporous aggregates, as illustrated. Once dissolution of the PAH compound occurs at the pore water-DNAPL interface, the PAH compound may diffuse into the bulk aqueous phase, where it becomes accoessible for degradation by microbes.

\section{Mineralization of I $^{14} \mathrm{C}$ Inaphthalene from coal tar in aqueous systems with silica}

All mineralization data have been corrected for background dpm. The dpm obtained from abiotic controls were consistently at background levels, indicating that the volatility of radiolabeled naphthalene did not significantly contribute to the measured activity.

Systems containing coal tar imbibed in silica did not contain a visible nonaqueous liquid phase at any time; instead, the coal tar remained held in the microporous silica for the duration of 
these studies. However, the aqueous phase in the biometer flasks turned a translucent gray color during the course of individual experiments lasting sixty days.

Two sets of experiments with [ ${ }^{14} \mathrm{C}$ ]naphthalene-labeled coal tar and silica were performed with slightly different results. In general, the mineralization achieved after 60 days in the first set of experiments was somewhat higher than in the second set of experiments under similar conditions ( $-30 \%$ cumulative mineralization vs. $\sim 20 \%)$. This difference is most likely attributable to variations in the initial viability of PAH-degrading culture used for the inocula. Data from the two sets of experiments are shown separately, although the trends exhibited by the data sets corroborate each other.

A biotic systems were used to establish that naphthalene mineralization was not occurring in the absence of added organisms (Figs. $2 \mathrm{~A}$ and $2 \mathrm{~B}$ ). Furthermore, it was found that the bioemulsifier-producing Acinetobacter calcoaceticus RAG-1 bacterium did not contribute to ${ }^{14} \mathrm{CO}_{2}$ evolution, indicating that PAH-degrading organisms were solely responsible for naphthalene mineralization (Figs. 2A and 2B). Control Masks inoculated only with PAH-degrading organisms yielded about $23 \%$ mineralization of labeled naphthalene after 10 days, $29 \%$ after 38 days, and $30 \%$ after 60 days in the first set of experiments. By day 60 mineralization was clearly increasing at a slow rate (Fig. 2A). The second set of experiments resulted in about 11\%, 18\%, and $21 \%$ cumulative mineralization after 13,38, and 60 days, respectively (Fig. 2B). These data are used as references for assessing the effects on naphthalene mineralization from addition of emulsan, RAG. 1 organisms, or cell-free culture broth.

Naphthalene mineralization in coal tar-silica systems did not change significantly upon the addition of cell-free culture broth from stationary-phase RAG-1 cultures, as shown in Figure 3A. The initial addition of $10 \mathrm{~mL}$ of this broth in the first set of experiments resulted in a response similar to that of the corresponding control flasks. Mineralization reached about $13 \%$ after 10 days, $25 \%$ after 38 days, and $29 \%$ by 60 days (Fig. 3A). In the second set of tests, mineralization was approximately $8 \%, 18 \%$, and $22 \%$ complete after 13,38 , and 60 days, respectively (Fig. 3B). Although mineralization in these systems was slower during the first two weeks than in the 
respective control flasks without broth, the average absolute difference from the controls after 60 days was less than $1 \%$ for both experiments. Systems receiving $10 \mathrm{~mL}$ of the broth after 45 days were similarly unaffected by the addition; in the first set of experiments, average mineralization reached $29 \%$ after 60 days (Fig. 3A). Doubling the volurne of broth added after 45 days produced no apparent effect, yielding $27 \%$ mineralization by day 60 (Fig. 3A).

Mineralization in coal tar-silica systems was also apparently unaffected by the addition of $0.015 \%$ emulsan, as shown in Figure 3B. The bioemulsifier was added either initially or after 45 days in the second set of experiments. Initial addition of emulsan resulted in an average of about $20 \%$ mineralization after 60 days, which was not significantly different from the control. The addition of emulsan at day $\mathbf{4 5}$ appeared to cause a slight increase in the mineralizatior curve, al though after 60 days mineralization was essentially the same as in the control.

The initial addition of various con-entrations of RAG-1 cells to coal tar-silica systems was investigated and found to produce no significant effect in either set of experiments. Figures $4 \mathrm{~A}$ and $4 \mathrm{~B}$ show the results of adding $2 \mathrm{~mL}$ inocula of $10^{10}, 10^{9}, 10^{8}$, or $10^{7} \mathrm{CFU} / \mathrm{mL}$ of stationaryphase RAG-1 culture to biometer flasks containing PAH-degrading organisms. Cumulative mineralization at the tenth day in the first set of experiments varied from a low value of about $17 \%$ to a high value of $24 \%$ for $10^{9}$ and $10^{7} \mathrm{CFU} / \mathrm{mL}$ RAG-1 inocula, respectively. The control flask containing only PAH-degrading organisms showed an intermediate value of 23\%. Mineralization at day 60 was highest in the $10^{7} \mathrm{CFU} / \mathrm{mL}$ RAG-1 system (36\%) and lowest in the control (30\%). The remaining RAG-1 systems exhibited 60-day mineralization that was only slightly higher in absolute terms than the control, with no apparent relationship between extent of mineralization and the number of cells added. The lack of a relationship between the addition of RAG-1 and an effect on naphthalene mineralization was also demonstrated in the second set of experiments (Fig. 4B), in which 60-day mineralization was slightly higher in the $10^{9} \mathrm{CFU} / \mathrm{mL}$ RAG-1 system (23\%), followed by the control (21\%) and the $10^{7}$ RAG-1 system (19\%).

Addition of stationary-phase RAG-1 cells 45 days after the start of an experiment showed results very similar to those for initial RAG-1 addition. No clear relationship between the 
concentration of cells added and naphthalene degradation was found, and no sudden increase in mineralization following RAG-1 addition was observed. Figure 5 shows the results of adding 2 $\mathrm{mL}$ of $10^{10}, 10^{9}, 10^{8}$, and $10^{7} \mathrm{CFU} / \mathrm{mL} \mathrm{RAG}-1$ inocula at day 45 in the first set of experiments. Mineralization after 60 days was approximately $28 \%$ complete in all except the $10^{10}$ case, which showed slightly higher mineralization ( $33 \%)$ even before inocul stion with RAG-1. Control flasks showed $-30 \%$ mineralization at day 60 ; thus, late addition of RAG-1 cells was ineffective in altering naphthalene mineralization in the first set of experiments. The addition of RAG-1 cells at 45 days was also tested in the second set of experiments (Fig. 4B). A slight increase in mineralization followed the addition of a $10^{9} \mathrm{CFU} / \mathrm{mL}$ inoculum of RAG-1 cells, although cumulative mineralization at day 60 (22\%) was effectively indistinguishable from that attained in the control nasks (21\%).

Mineralization of ${ }^{14} \mathrm{C}$ ]naphthalene from free-DNAPL coal tar in aqueous systems

This set of experiments evaluated systems without silica that contained coal tar as a free dense nonaqueous phase hiquid (DNAPL). The coal tar DNAPL coalesced into several globules at the bottom of biometer flasks. A certain amount of tar also formed thin layers at the surface of some biometer flasks over time, as described below. Inoculation with PAH-degrading microbes in systems containing silica was performed at the same time, and from the same culture used in the second set of experiments with silica, which accounts for the $-20 \%$ mineralization achieved in the control flasks described here.

Abiotic systems with free coal tar showed that naphthalene mineralization did not occur in the absence of added organisms, and biometer flasks inoculated only with RAG-1 bacteria also showed no mineralization. This demonstrated again that PAH-degrading bacteria were solely responsible for naphthalene mineralization. Mineralization in control flasks containing only PAH degrading organisms reached $18 \%$ after 59 days (Fig. 6). This is comparable to the controls in 
systems with coal tar imbibed in silica, which reached $21 \%$ mineralization by day 59 but did not plateau as quickly as in the systems with free-DNAPL tar.

Figure 7 shows the result of initially adding $0.015 \%$ emulsan to systems with free-DNAPL coal tar. The shape of the mineralization curve is very similar to that of the control, although mineralization is somewhat higher by day 59 compared to that in Figure 6 (22\%). Nonetheless, the corresponding mineralization in systems with coal tar imbibed into silica was $20 \%$, so neither the absence of silica nor the presence of emulsan seems to influence naphthalene degradation significantly.

The addition of RAG-1 cells was found to have little or no effect on mineralization over two months in systems containing free-DNAPL coal tar. RAG-1 inocula consisting of $2 \mathrm{~mL}$ of $10^{7}$ or $10^{9} \mathrm{CFU} / \mathrm{mL}$ of stationary-phase culture were added initially. After 59 days, mineralization had reached about $19 \%$ in the former case and $23 \%$ in the latter, which is not very different from the control or from corresponding tests with silica (Figs. 4B, 6, 8). The addition of a $10^{9}$ CFU/mL RAG-1 inoculum at day 45 resulted in a $5 \%$ increase in mineralization immediately after the addition, giving $26 \%$ naphthalene mineralization after 59 days. Unlike all other tests, the data shown for this case (Fig. 8) were obtained from only one biometer.

The tar in systems without silica remained a separate phase, with little or no emulsification being visually observed. After being added to the aqueous medium in biometer flasks, the tar settled as blobs at the bottom of the flasks. The tar blobs remained intact and resistant to flow during oscillatory shaking, and they frequently became attached to the flask bottom by a thin neck, which prevented the coal tar from freely moving about in the medium.

Several systems with free-phase coal tar DNAPL retained a clear, colorless, aqueous phase during the entire study. In these systems coal tar did not adhere to the flask in a ring along the flask wall, nor was it present as a film at the surface. Other biometer flasks developed a ring-like accumulation of coal tar on the flask wall over time, and in these instances the aqueous medium turned a translucent gray color after several weeks. Surface layers of tar were present in varying degrees, from being absent to forming a thin oily sheen to a brown film that nearly covered the 
interface between air and water. However, surface films or tar rings on glass did not appear to be related to the contents of flasks. For example, of the biometer flasks with clear aqueous medium and no tar rings or films, one contained only PAH-degrading organisms and the other held RAG-1 bacteria as well. Three other flasks contained a tar ring, grayish medium and a sparse surface film: one contained PAH-degrading organisms, the second held PAH-degrading organisms and RAG-1, and the third had PAH-degrading bacteria and $0.015 \%$ emulsan. The differences in the appearance of $\operatorname{tar}$ in these systems is due to unknown factors.

\section{DISCUSSION}

The results presented here indicate that the bioemulsifier emulsan and two related fractions of Acinetobacter calcoaceticus RAG-1 cultures were ineffective in altering naphthalene mineralization from coal tar in the aqueous systems studied. Cell-free culture broth from RAG-1 cultures, stationary-phase RAG-1 inocula of various concentrations, or $0.015 \%$ emulsan was added to systems containing $\left[{ }^{14} \mathrm{C}\right]$ naphthalene-labeled coal tar and PAH-degrading bacteria that had been isolated from contaminated soils and wastes. Coal tar in these systems was either imbibed in rnicroporous silica or present as a free dense nonaqueous phase liquid (DNAPL). Naphthalene mineralization was determined from ${ }^{14} \mathrm{CO}_{2}$ evolution and was compared to control systems receiving PAH-degrading bacteria but none of the abovementioned additions. Napthalene mineralization in these studies was neither enhanced nor inhibited by the presence of emulsan, RAG-1 cells, or RAG-1 cell-free broth.

Two sets of experiments were performed under similar conditions with slightly different results. The first set of experiments resulted in approximately $30 \%$ mineralization of naphthalene after 60 days, while the second set of tests yielded about $20 \%$ mineralization over 60 days. This difference was probably a result of variations in the PAH-degrading culture used for the inocula. Nevertheless, the trends shown in both experiments corroborate each other.

The addition of cell-free broth, emulsan, or RAG-1 inocula late in the experiments did not give significantly different results from initial additions of these substances. The concentration of 
RAG-1 inocula was not correlated to any change in naphthalene mineralization, nor was the response dependent on whether coal tar was free-phase DNAPL or imbibed within microporous silica. Coal tar imbibed into microporous silica remained entrapped within the silica throughout the experiments and was never present as a free phase liquid. Tar in systems without silica existed as a separate liquid phase without observable emulsification, despite the fact that the tar contained 1methylnaphthalene, which has been shown to enhance slightly the emulsification of naphthalene and other aromatic compounds by emulsan [22]. In some cases free-phase tar formed ring-like accumulations on flask walls or films of various thickness at the water-air interface, but these phenomena were apparently unrelated to the contents of the systems.

The very slow rate of naphthalene mineralization observed after $\mathbf{4 0}$ days in most systems is not explained by these tests. Perhaps mass transport of naphthalene from coal tar into the aqueous medium limits biodegradation. Free-phase coal tar in experimental systems without silica existed as large globs at the bottom of flasks, with relatively little interfacial area for mass transfer to occur over. In systems with silica, the sorption of tar onto the pore walls and outer surfaces of silica particles may retard mass transport and biodegradation [10]. Another possible explanation is the formation of a semi-rigid skin or film of coal tar that may impede mass transfer across the DNAPLwater interface [3]. A third explanation for the decline in naphthalene mineralization could be the divergent metabolic pathways of the PAH-degrading pseudomonads used in this study. Although the broad-based enzymes of Pseudomonas species allow these organisms to degrade many natural organic compounds and xenobiotics, "dead-end" intermediates can form when enzymes for subsequent degradation steps are not available [7]. Perhaps the PAH-degrading bacteria transformed naphthalene to intermediates that could not be mineralized. However, this is not reported for naphthalene [10], and further, the recovery of dpm from the aqueous phase of systems at the end of this and related studies indicates that if such compounds were formed, they were only sparingly soluble in water. Aqueous phase dpm values were not significantly greater than those expected for naphthalene alone. Whether or not the above explanations are sufficient to account for the decline in mineralization is unknown. Work is in progress to assess the rate of mass transfer of 
naphthalene to the aqueous phase from coal tar imbibed into microporous silica for both freshlyprepared and aged samples.

It is unclear why emulsan, cell-free RAG-1 broth or living RAG-1 cells had no effect on naphthalene emulsification and mineralization. Emulsan was found to inhibit aromatic mineralization from crude oil by a mixe:d PAH-degrading culture in a previous study [20], but because inhibition was not observed from emulsan or cell-free culture broth in these experiments, interactions between emulsan and PAH-degrading bacteria may be species-specific. The intact RAG-1 cells incorporated into systems with coal tar were in the stationary growth phase, and they should have lost most of their cell-associated emulsan prior to being added. The absence of cellassociated emulsan would have given the cells a higher affinity for adherence and growth on hydrocarbons [19]. However, RAG-1 cells spread on agar plates with naphthalene as the sole carbon source did not grow, so Acinetobacter calcoaceticus may not have been capable of producing emulsan in these experiments. Finally, the coal tar used in these experiments contained no identifiable aliphatic compounds, and thus conditions for PAH emulsification by emulsan were probably not optimum.

\section{REFERENCES}

1. Elliott, M. A. and G. R. Yohe. 1981. The coal industry and coal research and development in perspective. In M. A. Elliot (ed.), Chemistry of Coal Utilization. John Wiley and Sons, New York, pp. 1-54.

2. MeNeil, D. 1981. High-temperature coal tar. In M. A. Elliott (ed.), Chemistry of Coal Utilization. John Wiley and Sons, New York, pp. 1003-1083.

3. Luthy, R. G., A. Ramaswami, S. Ghoshal and W. Merkel. 1993. Interfacial films in coal tar-DNAPL/water systems. Submitted for publication. 
4. Laha, S. and R.C. Luthy. 1992. Effects of nonionic surfactants on the solubilization and mineralization of phenanthrene in soil-water systems. Biotechnol. Bioeng. 40:1367-1380.

5. Jain, D. K., H. Lee and J. T. Trevors. 1992. Effect of addition of Pseudomonas aeruginosa UG2 inocula or biosurfactants on biodegradation of selected hydrocarbons in soil. J. Industrial Microbiol. 10:87-93.

6. Mueller, J. G., P. J. Chapman and P. H. Pritchard. 1989. Creosotecontaminated sites. Environ Sci. Technol. 23:1197-1201.

7. Golovleva, L., O. V. Maltseva and I. P. Solyanikova. 1992. Metabolism of foreign compounds in Pseudomonas spp. In E. Galli, S. Silver, and B. Witholt (ed.), Pseudomonas, Molecular Biology and Biotechnology. American Society for Microbiology, Washington, D.C., pp. 231-238.

8. Goswami, P. and H. D. Singh. 1991. Different modes of hydrocarbon uptake by two Pseudomonas species. Biotechnol. Bioeng. 37:1-11.

9. Laha, S. and R. G. Luthy. 1991. Inhibition of phenanthrene mineralization by nonionic surfactants in soil-water systems. Environ. Sci. Technol. 25:1920-1930.

10. Bouwer, E. J. and A. J. B. Zehnder. 1992. Bioremediation of organic compounds - putting microbial metabolism to work. Department of Geography and Environmental Engineering, The Johns Hopkins University, Baltimore, MD.

11. Guerin, W. F. and S. A. Boyd. 1992. Differential bioavailability of soil-sorbed naphthalene to two bacterial species. Appl. and Environ. Microbiol. 58:1142-1152.

12. Llu, Z., S. Laha and R. G. Luthy. 1991. Surfactant solubilization of polycyclic aromatic hydrocarbon compounds in soil-water suspensions. Wat. Sci. Tech. 23:475485.

13. Hayes, M. E., E. Nestaas and K. R. Hrebenar. 1986. Microbial surfactants. Chemtech. 16:239-243. 
14. Mulligan, C. N., G. Mahmourides and B. F. Gibbs. 1989. The influence of phosphate metabolism on biosurfactant production by Pseudomonas aeruginosa. J. Biotechnol. 12:199-210.

15. Goldman, S., Y. Shabtai, C. Rubinovitz, E. Rosenberg and D. L. Gutnick. 1982. Emulsifier in Acinetobacter calcoaceticus RAG-1: distribution of cell-free and cell-associated cross-reacting material. Appl. Environ. Microbiol. 44:165170.

16. Shabtai, Y. and D. L. Gutnick. 1985. Exocellular esterase and emulsan release from the cell surface of Acinetobacter calcoaceticus. J. Bacteriol. 161:1176-1181.

17. Zuckerberg, A., A. Diver, Z. Peeri, D. L. Gutnick and E. Rosenberg. 1979. Emulsifier of Arthrobacter RAG-1: chemical and physical propentes. Appl. Environ. Microbiol. 37: 414-420.

18. Rosenberg, E., A. Zuckerberg, C. Rubinovitz and D. L. Gutnick. 1979. Emulsifier of Arthrobacter RAG-1: isolation and emulsifying properties. Appl. Environ. Microbiol. 37:402-408.

19. Rosenberg, E., N. Kaplan, O. Pines, M. Rosenberg and D. Gutnick. 1983. Capsular polysaccharides interfere with adherence of Acinetobacter calcoaceticus to hyd xcarbon. FEMS Microbiol. Lett. 17:157-160.

20. Foght, J. M., D. L. Gutnick and D. W. S. Westlake. 1989. Effect of emulsan on biodegradation of crude oil by pure and mixed bacterial cultures. Appl. Environ. Microbiol. 55:36-42.

21. Zosim, Z, D. Gutnick and E. Rosenberg. 1982. Properties of hydrocarbon-inwater emulsions stabilized by Acinetobacter RAG-1 emulsan. Biotechnol. Bioeng. 24:281-292.

22. Rosenberg, E., A. Perry, D. T. Gibson and D. L. Gutnick. 1979. Emuisifier of Arthrobacter RAG-1: specificity of hydrocarbon substrate. Appl. Environ. Microbiol. $37: 409-413$ 
23. Litchneld, C. D. (Environmental Technology Applications, Monroeville, PA) 1992. Personal communication.

24. Rosenberg, E., A. Gottlieb and M. Rosenberg. 1983. Inhibition of bacterial adherence to hydrocarbons and epithelial cells by emulsan. Infection and Immunity. 39: 1024-1028.

25. American Public Health Association. 1980. Standard Methods for the Examination of Water and Wastewater, 15th ed. American Public Health Association, Washington, D.C.

26. Peters, C. 1992. Ph.D. thesis. Carnegie Mellon University, Pittsburgh, PA.

27. Ramaswami, A. and R. G. Luthy. 1993. Modeling dissolution and biodegradation of naphthalene from coal tar in microporous media. Manuscript in preparation. Department of Civil Engineering, Camegie Mellon University, Pittsburgh, PA. 
TABLE 1. Ten major identifiable components of Stroudsburg, PA coal tar [26]

\begin{tabular}{|lcc|}
\hline & Molecular & Weight Percent \\
\cline { 2 - 3 } methylchrysenes & Weight & \\
trialkylated naphthalenes & 170 & 4.4 \\
methylpyrene & 216 & 4.3 \\
1-methylnaphthalene & 142 & 3.9 \\
2-methylnaphthalene & 142 & 3.80 \\
dimethylphenanthrene & 206 & 3.75 \\
naphthalene & 128 & 2.3 \\
phenanthrene & 178 & 2.16 \\
2,6-dimethylnaphthalene & 156 & 2.12 \\
2-ethylnaphthalene & 156 & 1.99 \\
\hline totala & & 30.56 \\
\hline
\end{tabular}

The total weight percent of all identifiable compounds was about 48\% [26]. 


\section{FIGURE LEGENDS}

Figure 1. The processes through which dissolution, mass transfer and biodegradation of PAH compounds from coal tar dense nonaqueous phase liquid (DNAPL) imbibed in microporous media is envisioned to occur [27].

Figure 2. Naphthalene mineralization in aqueous systems containing coal tar imbibed in microporous silica in first set (A) and second set (B) experiments. Data are reported as the percent of ${ }^{14} \mathrm{C}$-labeled naphthalene mineralized. In this and subsequent figures, inocula of $2 \mathrm{~mL}$ were used and PAH-degrading inocula were taken from a $10^{7} \mathrm{CFU} / \mathrm{mL}$ culture. Neither the abiotic control nor those systems containing only bioemulsifier-producing RAG-1 bacteria exhibited naphthalene mineralization, indicating that PAH-degrading bacteria were solely responsible for ${ }^{14} \mathrm{CO}_{2}$ evolution. Data points are the average of several tests, and error bars represent one standard deviation.

Figure 3. Naphthalene mineralization in first set (A) and second set (B) experimental systems containing coal tar imbibed in microporous silica, with PAH-degrading inocula, and cell-free RAG-1 culture broth or $0.015 \%$ emulsan. Cell-free broth was obtained from stationary-phase RAG-1 cultures. The control systems contained PAH-degrading bacteria but neither broth nor emulsan.

Figure 4. Naphthalene mineralization in first set (A) and second set (B) experimental systems containing coal tar imbibed in microporous silica with PAH-degrading inocula, and additions of RAG-1 inocula at concentrations from $10^{7}$ to $10^{10} \mathrm{CFU} / \mathrm{mL}$ 
Figure 5. Naphthalene mineralization in first set experimental systems containing PAH-degrading bacteria and coal tar imbibed in microporous silica. RAG-1 inocula at concentrations from $10^{7}$ to $10^{10}$ were added at 45 days.

Figure 6. Naphthalene mineralization in second set experimental systems containing coal tar as a free dense nonaqueous phase liquid (DNAPL). Neither the abiotic control nor those systems containing only RAG-1 bacteria exhibited naphthalene mineralization, indicating that PAHdegrading organisms were solely responsible for ${ }^{14} \mathrm{CO}_{2}$ evolution.

Figure 7. Naphthalene mineralization in second set experimental systems with free-phase coal tar and $0.015 \%$ added emulsan. The controls contained PAH-degrading inocula only.

Figure 8. Naphthalene mineralization in second set experimental systems with free-phase coal tar and additions of RAG-1 cells at the times shown. 


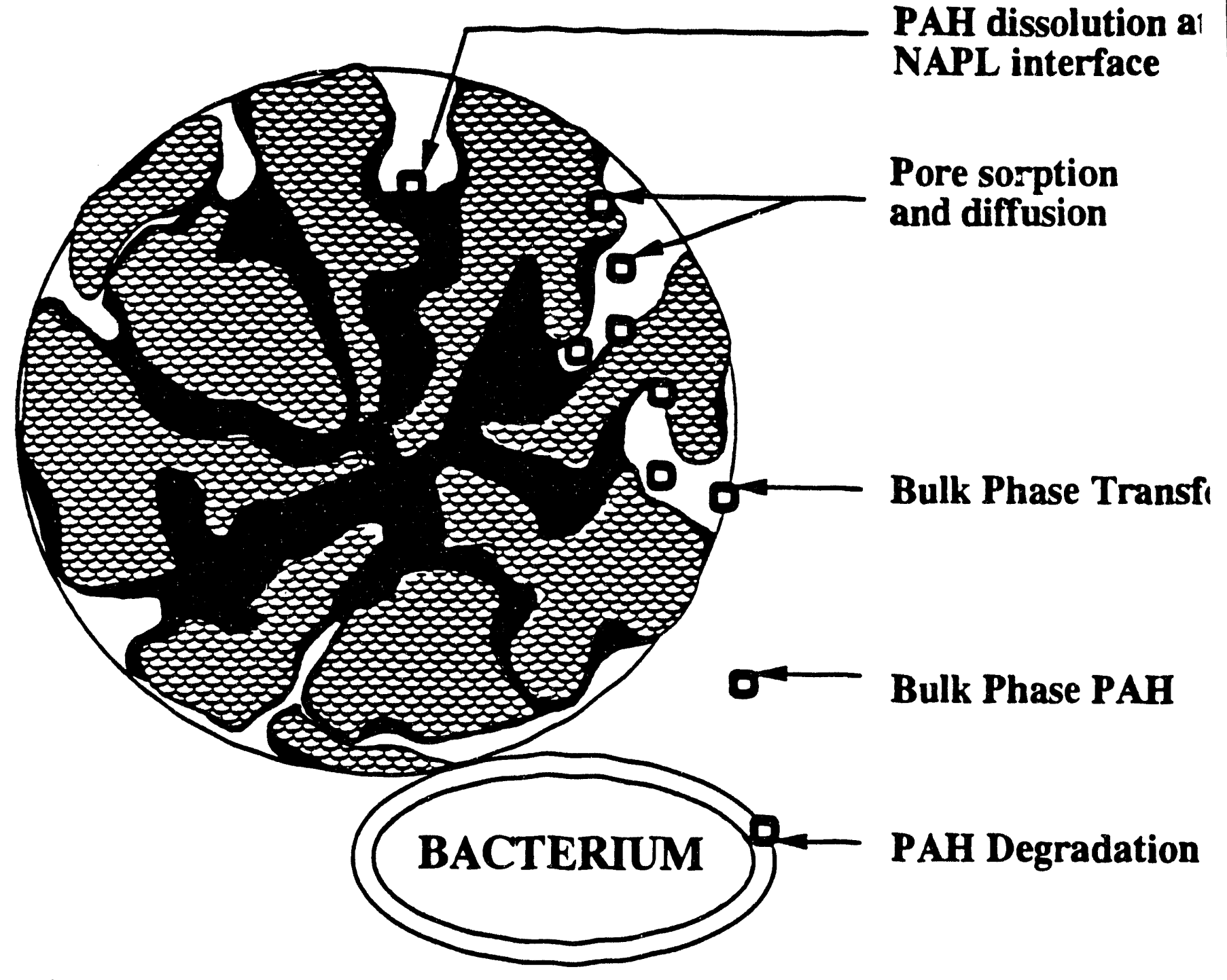

Figure 1. Karen L. Skubal and Richard G. Luthy. 

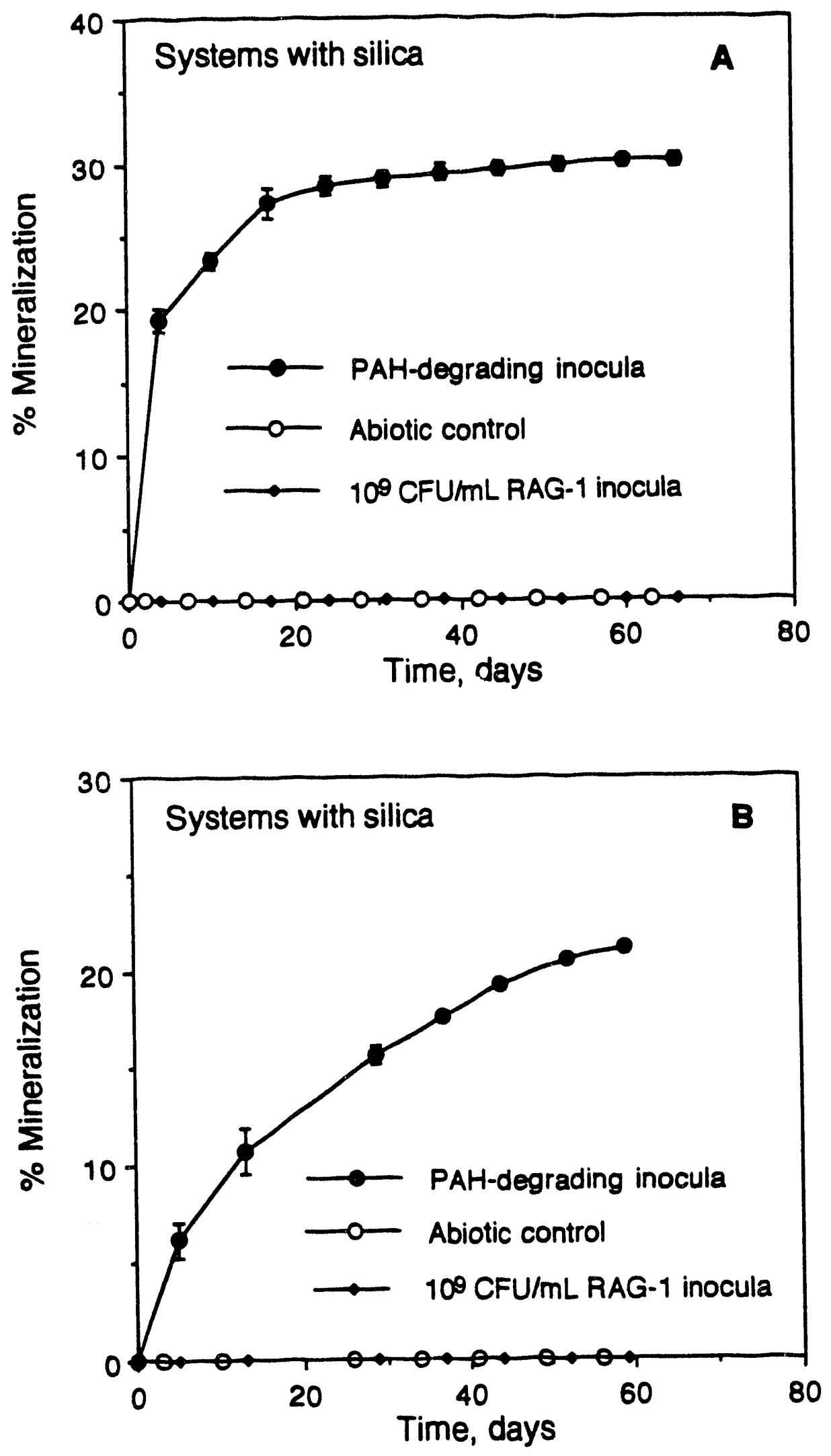

Figure 2 Karen L. Skubal and Richard G. Luthy. 

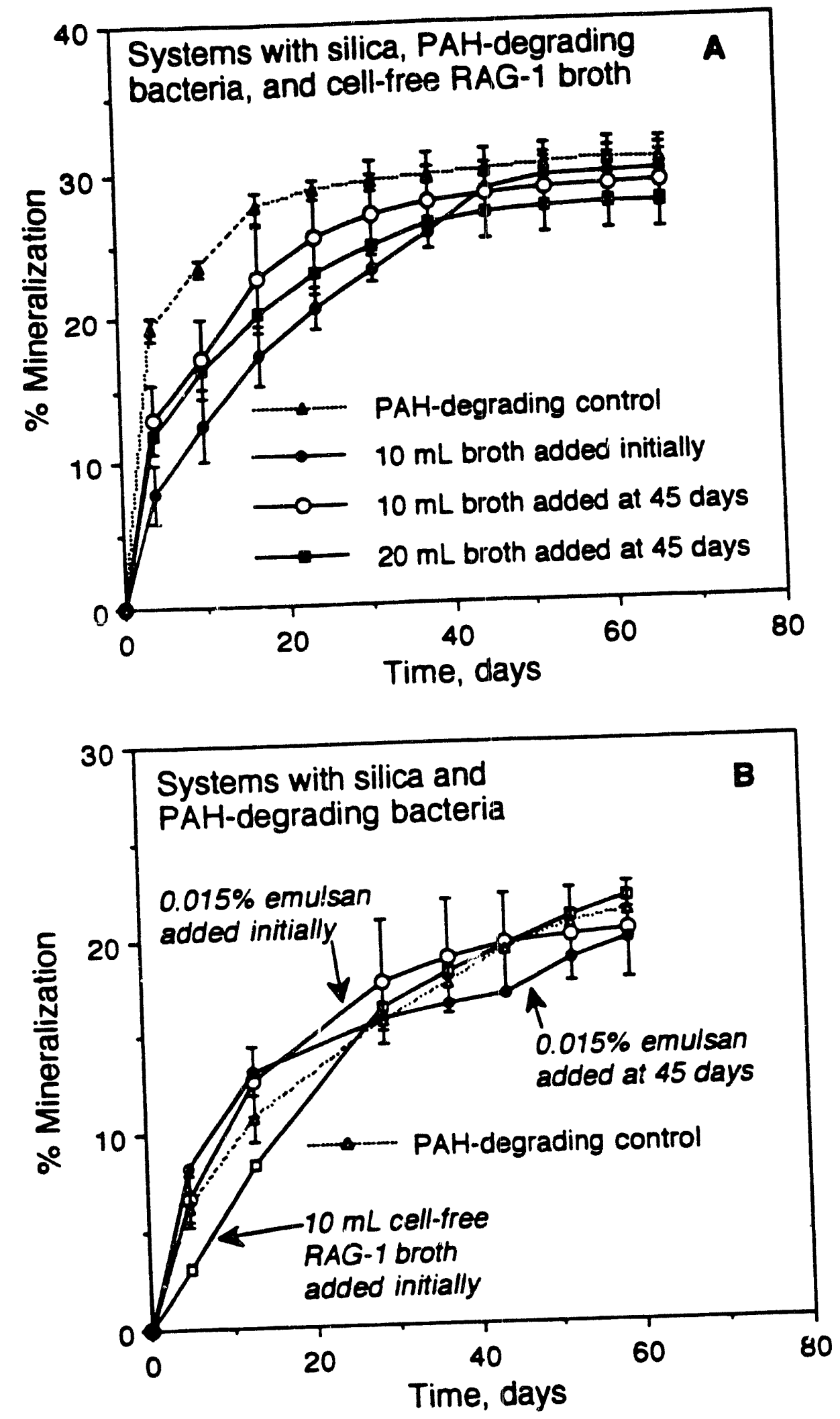

Figure 3. Karen L Skubal and Richard G. Luthy 

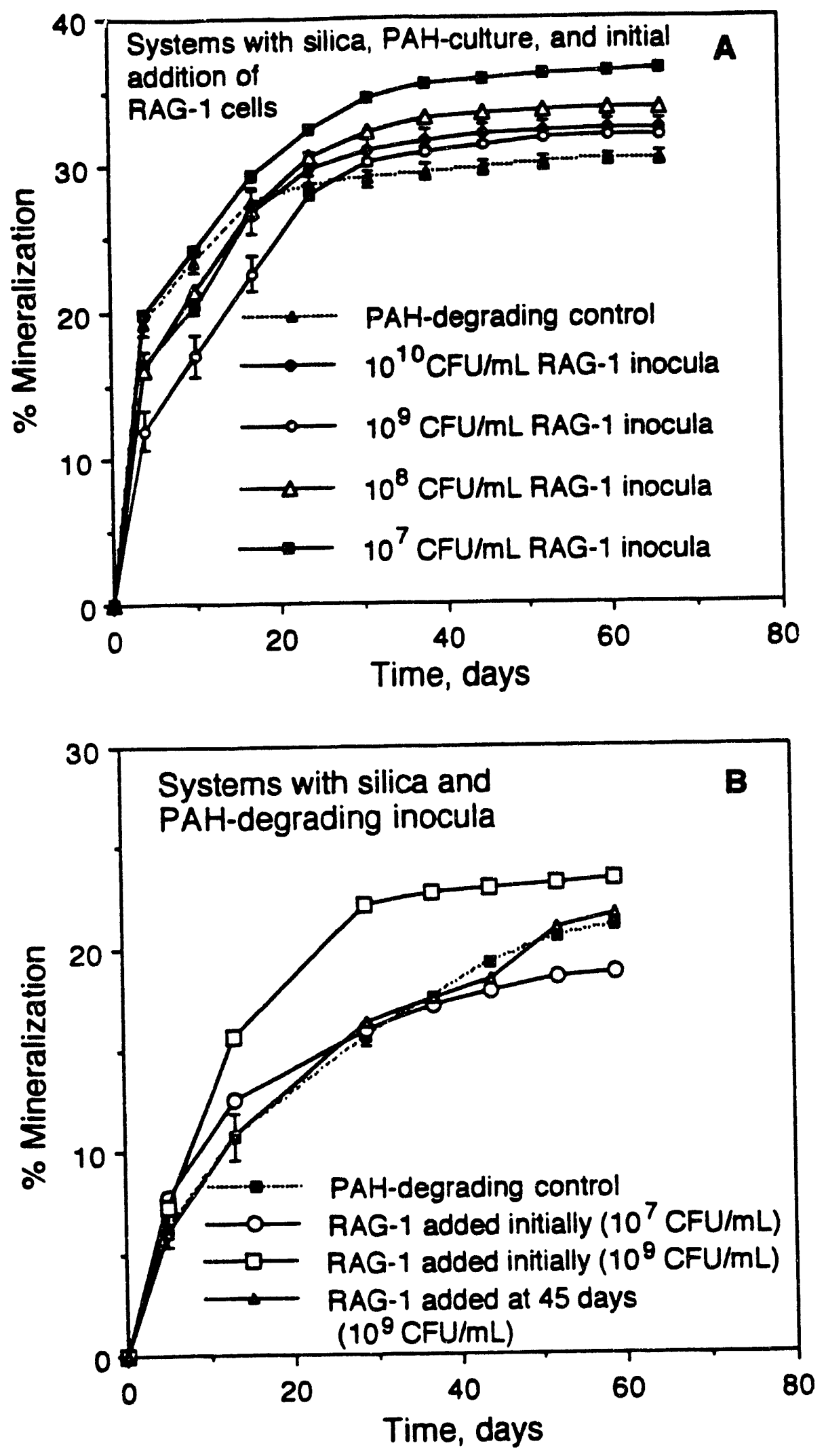

Figure 4. Karen L. Skubal and Richard G. Luthy 


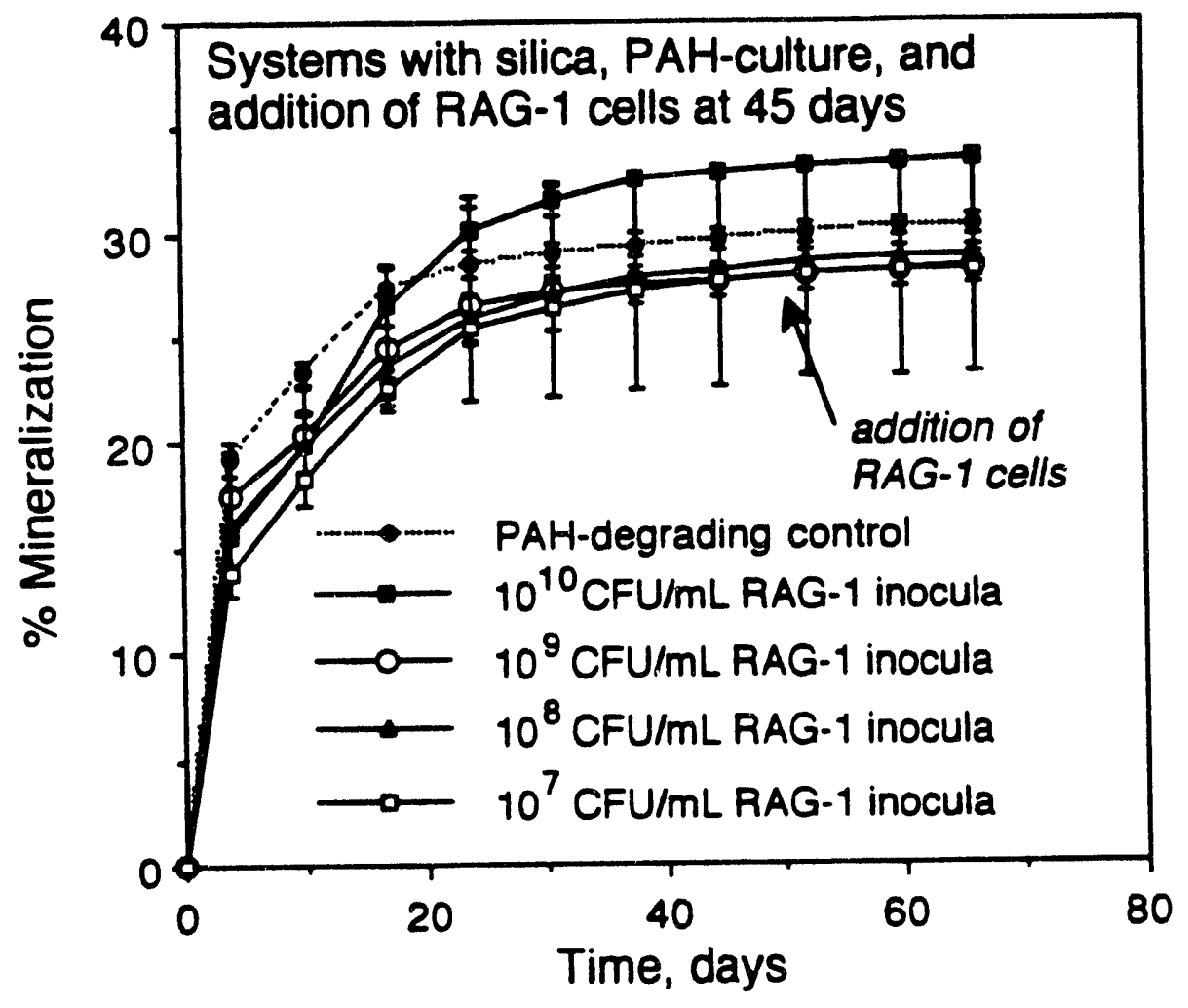

Figure 5. Karen L Skubal and Richard G. Luthy 


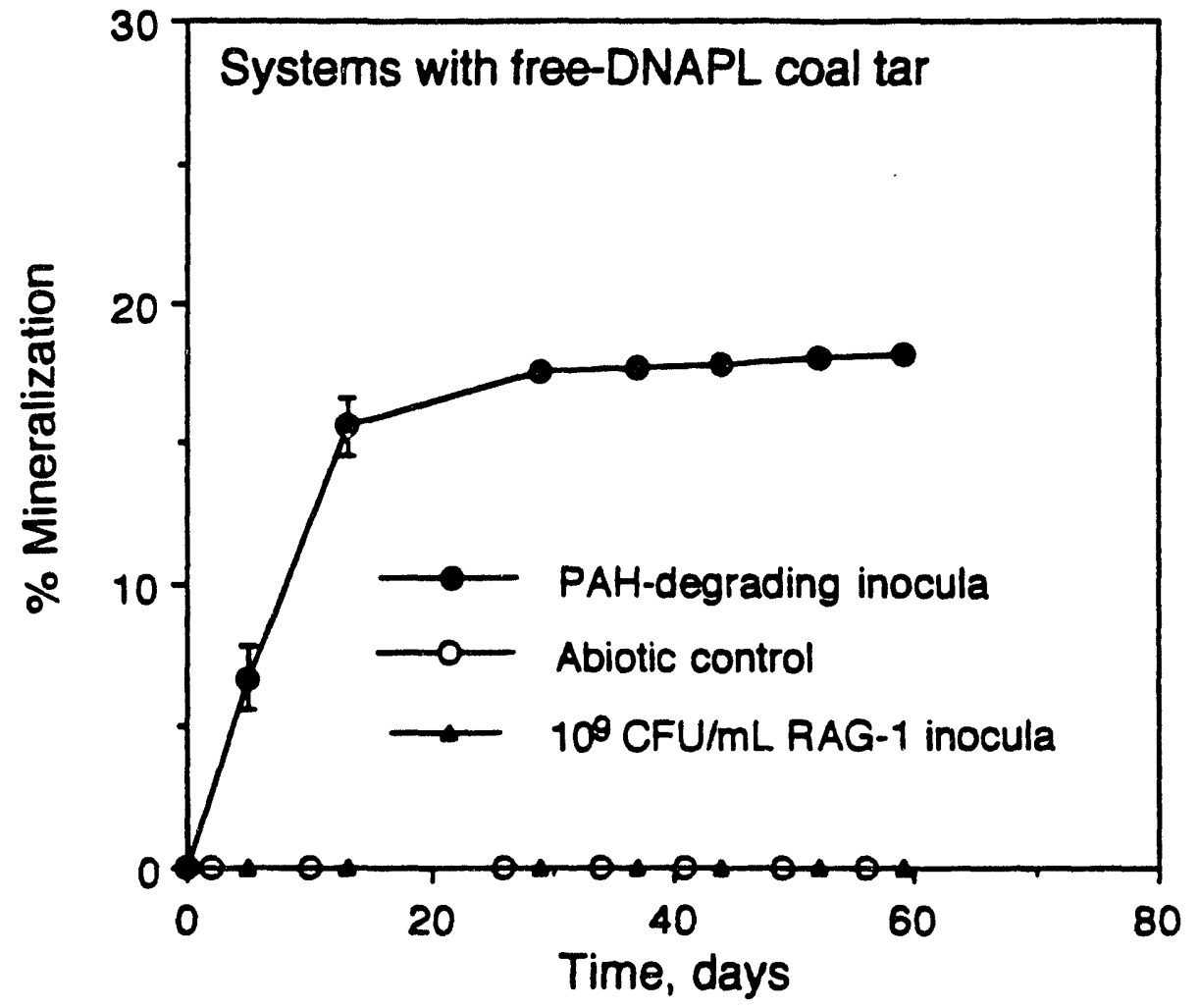

Figure 6. Karen L. Skubal and Richard G. Luthy. 


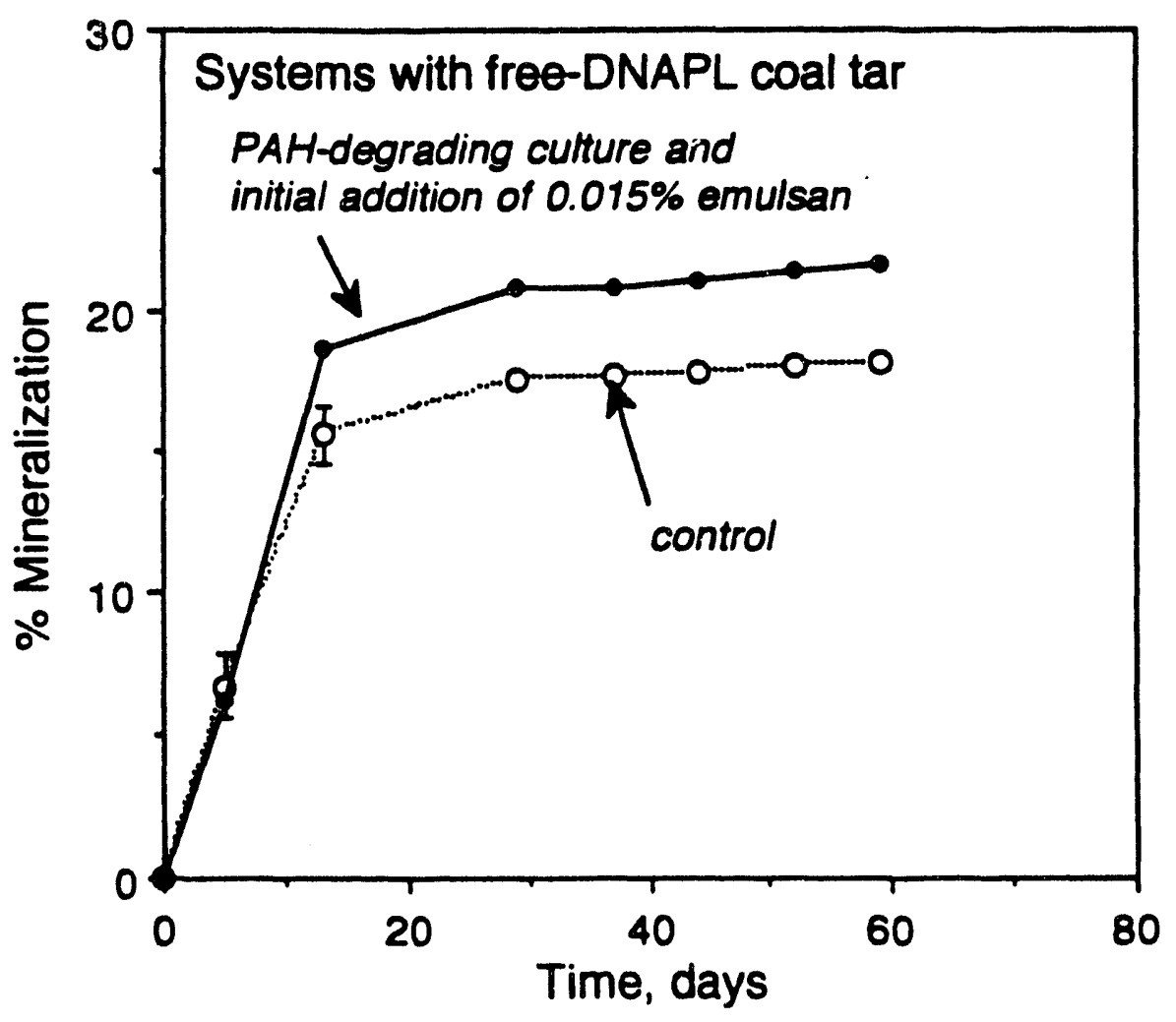

Figure 7. Karen L Skubal and Richard G. Luthy 


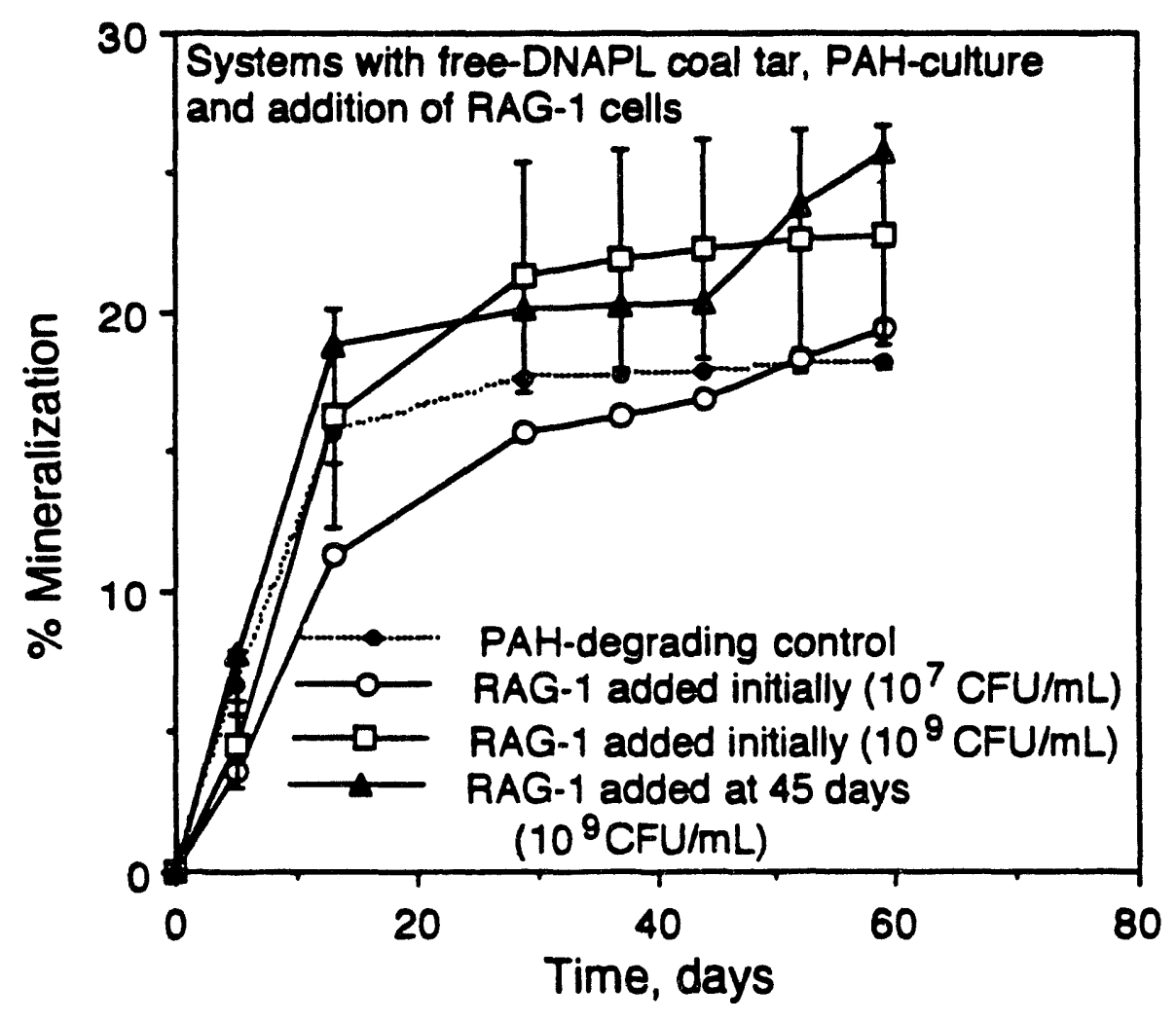

Figure 8. Karen L Skubal and Richard G. Luthy 

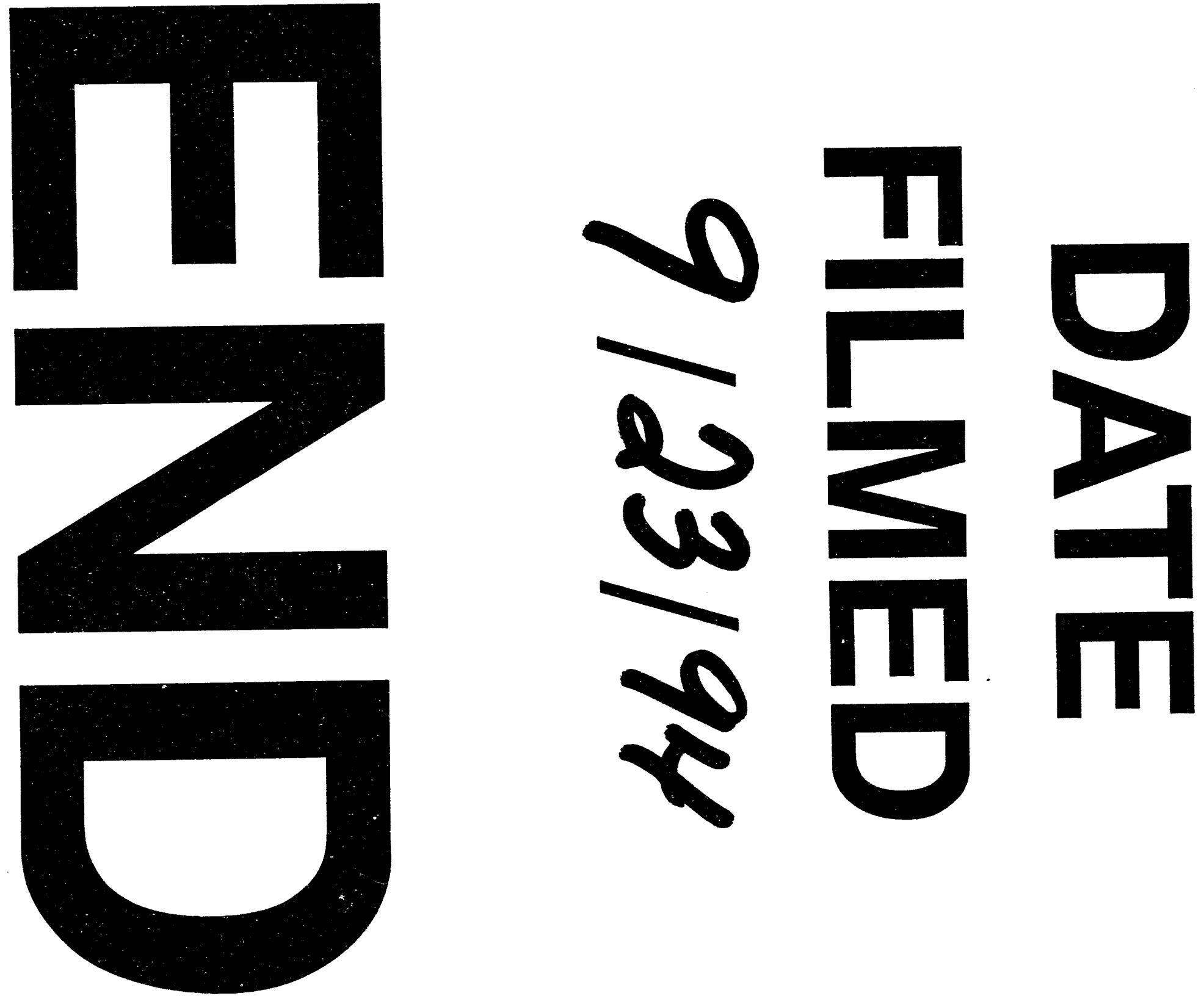
L 